\title{
COMPARAÇÃO ENTRE MÉTODOS DE QUANTIFICAÇÃO EM CROMATOGRAFIA GASOSA: UM EXPERIMENTO PARA CURSOS DE QUÍMICA
}

\author{
Carolina Bastos Pereira Ligiero, Lindomar Augusto dos Reis, Gabrieli Lessa Parrilha, Milton Baptista Filho e Maria \\ Cristina Canela* \\ Centro de Ciência e Tecnologia, Universidade Estadual do Norte Fluminense Darcy Ribeiro, Av. Alberto Lamego, 2000, 28013 - \\ 602 Campos dos Goytacazes - RJ, Brasil
}

Recebido em 23/4/08; aceito em 3/2/09; publicado na web em 7/5/09

\begin{abstract}
COMPARISON OF QUANTIFICATION METHODS IN GAS CHROMATOGRAPHY: AN EXPERIMENT FOR CHEMISTRY COURSES. This article describes an experiment designed to teach quantitative determination in gas chromatography (GC) in Organic and Analytical Chemistry practical classes. The experiment consisted of extracting and analyzing eugenol from clove seeds to perform a quantitative approach aimed at comparing results obtained by external and internal calibration procedures. Therefore, this experiment proved to be very effective tool to enhance students awareness on the need to understand different types of calibration in GC and on how to avoid common experimental errors, and to find the best ways to eliminate their interference during the quantitative analysis phase.
\end{abstract}

Keywords: GC; quantitative analysis; teaching.

\section{INTRODUÇÃO}

A cromatografia gasosa é um tópico apresentado nos cursos de Química, tanto nas disciplinas de Química Analítica como de Química Orgânica. No primeiro caso, a cromatografia gasosa é mostrada na maioria das vezes como uma ferramenta analítica quantitativa, uma vez que o cromatógrafo está ligado a um detector capaz de responder a um determinado composto de maneira proporcional a sua concentração. ${ }^{1,2}$ No caso da Química Orgânica, um enfoque maior é dado ao aspecto qualitativo e de separação de misturas, embora a sua utilização em termos quantitativos também ocorra. A cromatografia gasosa é normalmente utilizada como ferramenta analítica nos laboratórios de Química Orgânica durante o curso de graduação. ${ }^{3}$ Os livros de Química Orgânica, entretanto, raramente trazem teorias sobre o assunto, apenas ressaltando um dos detectores utilizados que é o espectrômetro de massas. ${ }^{4}$

A difusão e o desenvolvimento da cromatografia gasosa se deram por seu potencial para realizar análises quantitativas com velocidade e rapidez na determinação de compostos orgânicos presentes em concentrações relativamente baixas em diferentes tipos de amostras. As matrizes complexas normalmente sofrem diferentes processos de extração e o composto de interesse pode ser então quantificado através desta técnica, que passa assim a ter grande importância na pesquisa científica básica e nas suas aplicações na indústria.

O método direto para análises cromatográficas quantitativas envolve a preparação de uma série de soluções-padrão de composições próximas à concentração do analito na amostra, com o objetivo de realizar uma calibração externa. Os cromatogramas dos padrões são então obtidos, e as alturas ou as áreas dos picos terminam lançados em um gráfico em função da concentração. Idealmente a curva obtida deve ser uma reta que passa pela origem e a concentração da amostra é obtida a partir da equação desta reta. ${ }^{5}$ Porém, algumas vezes se obtêm curvas com coeficientes lineares positivos ou negativos, cabendo ao analista a realização de um tratamento criterioso dos dados da curva. As fontes mais importantes de erros analíticos através do uso deste método são as incertezas no volume da amostra e na preparação dos padrões, além de problemas durante o processo de injeção. As amostras são normalmente pequenas e as incertezas associadas à injeção de um volume reprodutível deste tamanho com

*e-mail: mccanela@uenf.br uma microsseringa podem chegar a um erro relativo alto. ${ }^{5}$

Outro método que pode ser utilizado para análises quantitativas com maior precisão é a padronização interna. Nesta metodologia podem ser minimizadas as incertezas introduzidas na injeção da amostra devido às pequenas quantidades de amostras injetadas e diferentes analistas. É especialmente indicada para técnicas com pouca reprodutibilidade, como no caso das injeções manuais. ${ }^{6} \mathrm{Na}$ padronização interna uma quantidade de uma substância medida cuidadosamente, que atua como padrão interno, é introduzida em cada padrão e na amostra, e a razão entre as áreas do pico do analito e do padrão interno funcionam como parâmetro analítico. ${ }^{3,5} \mathrm{Na}$ escolha de um composto como padrão interno deve-se observar que o pico referente ao padrão esteja bem resolvido dos demais picos dos componentes da amostra e elua próximo ao sinal do analito. O padrão interno deve, ainda, ser altamente puro, não reagir com nenhum dos componentes da amostra e ser quimicamente similar ao analito de interesse. ${ }^{6}$

Além da escassa disponibilidade de propostas de experimentos utilizando cromatografia gasosa em Química Orgânica na literatura, é mais raro ainda encontrar experimentos com uma abordagem quantitativa que ressaltem a função da padronização interna nesta técnica instrumental. ${ }^{7}$

Este trabalho propõe a realização de um experimento para ser ministrado nas disciplinas de laboratório de Química Orgânica ou Analítica com o objetivo de oferecer aos seus participantes aprendizado sobre cromatografia gasosa e ainda sobre como conduzir comparações entre a qualidade dos resultados obtidos com os diferentes métodos quantitativos. Além disso, pretende mostrar como o erro de injeção através de diferentes analistas pode influenciar no resultado final de uma análise quantitativa. Neste caso, o método de experimentação envolve a quantificação do teor de eugenol no óleo extraído de cravo-da-índia através dos métodos de calibração direta e padrão interno.

\section{PARTE EXPERIMENTAL}

\section{Obtenção do óleo de cravo-da-índia}

O óleo essencial foi obtido através da utilização de $16 \mathrm{~g}$ de cravo-da-índia e $200 \mathrm{~mL}$ de água destilada pelo método de destilação por arraste de vapor (Figura 1). A mistura foi colocada em 
um balão de fundo redondo com pérolas de vidro, adaptado a um condensador com um béquer para coleta do destilado. O líquido do balão foi aquecido em uma manta aquecedora até a ebulição. $\mathrm{O}$ produto obtido apresentou-se como uma emulsão (água misturada ao óleo de cravo). O arraste a vapor foi finalizado quando o volume no balão atingiu cerca de $50 \mathrm{~mL}$. O líquido resultante foi extraído em um funil de separação com 3 porções de $50 \mathrm{~mL}$ de diclorometano (Vetec). As três porções orgânicas foram então reunidas, secadas com sulfato de sódio (Syntht) e submetidas à evaporação em evaporador rotatório, fornecendo então $1,94 \mathrm{~g}$ de óleo de cravo (12\%).

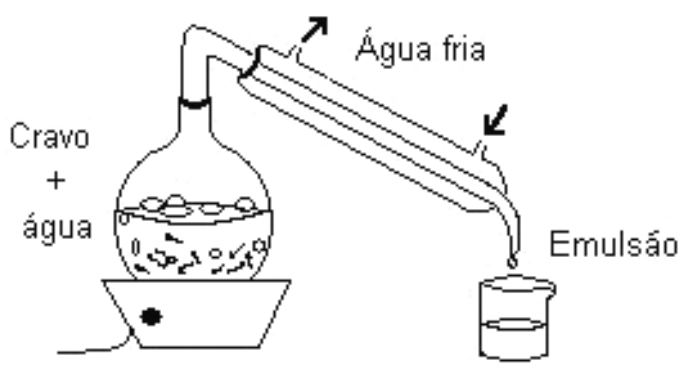

Figura 1. Aparato experimental para extração por arraste de vapor

\section{Preparo dos padrões para as curvas de calibração}

Foram confeccionadas cinco soluções-padrão com diferentes concentrações de eugenol obtido comercialmente (Firmenich) em $25 \mathrm{~mL}$ de diclorometano (Vetec). Como padrão interno adicionou-se uma quantidade fixa de $46 \mathrm{mg}\left(1,84 \mathrm{~g} \mathrm{~L}^{-1}\right)$ de carvona (Firmenich) a cada padrão. As massas e concentrações de eugenol em cada padrão são mostradas na Tabela 1 .

Tabela 1. Preparação dos padrões

\begin{tabular}{lcc}
\hline Padrão & Massa de eugenol (mg) & $\begin{array}{c}\text { Concentração de eugenol } \\
\left(\mathrm{g} \mathrm{L}^{-1}\right)\end{array}$ \\
\hline 1 & 20,8 & 0,83 \\
2 & 31,2 & 1,25 \\
3 & 52,0 & 2,08 \\
4 & 72,8 & 2,91 \\
5 & 93,6 & 3,74 \\
\hline
\end{tabular}

\section{Preparo da amostra analítica}

Uma alíquota de $51 \mathrm{mg}$ da amostra do óleo essencial extraída do cravo-da-índia foi diluída para $25 \mathrm{~mL}$ de diclorometano. Como padrão interno foram adicionados $46 \mathrm{mg}$ de carvona, conforme feito anteriormente com os padrões.

\section{Injeção das amostras}

As injeções das soluções-padrão para a construção das curvas de calibração foram feitas por um mesmo analista. A amostra de óleo de cravo-da-índia foi injetada por quatro analistas diferentes. Os cromatogramas obtidos foram integrados e as curvas de calibração foram feitas utilizando-se as áreas dos picos.

\section{Condições cromatográficas}

As análises cromatográficas foram realizadas em um cromatógrafo a gás acoplado a um detector de ionização em chama (CG-DIC) modelo Shimadzu 17A, utilizando uma coluna DB-1 J\&W de $30 \mathrm{~m}$, diâmetro interno $0,25 \mathrm{~mm}$ e espessura do filme de $0,25 \mu \mathrm{m}$. A temperatura do injetor e do detector foram mantidas em 200 e $230{ }^{\circ} \mathrm{C}$, respectivamente, e a programação do forno foi de 50 até $230^{\circ} \mathrm{C}$ com uma taxa de aquecimento de $15^{\circ} \mathrm{C} \mathrm{min}^{-1}$. O gás carregador utilizado foi o hidrogênio com uma vazão na coluna de $1,8 \mathrm{~mL} \mathrm{~min}^{-1}$. O volume de injeção foi de $1 \mu \mathrm{L}$.

\section{Tratamento dos dados}

Os tratamentos estatísticos e a geração dos gráficos foram feitos no software Origin 7.

\section{RESULTADOS E DISCUSSÃO}

A Figura 2 mostra o cromatograma obtido a partir da injeção de um dos padrões. Os sinais com tempos de retenção de 5,32 e 6,29 min referem-se à carvona (padrão interno) e ao eugenol, respectivamente. É possível observar na figura que a resolução obtida permitiu a separação completa dos dois componentes, não havendo sobreposição dos picos cromatográficos. Os valores obtidos para as áreas dos padrões e a razão entre as áreas são apresentados na Tabela 2.

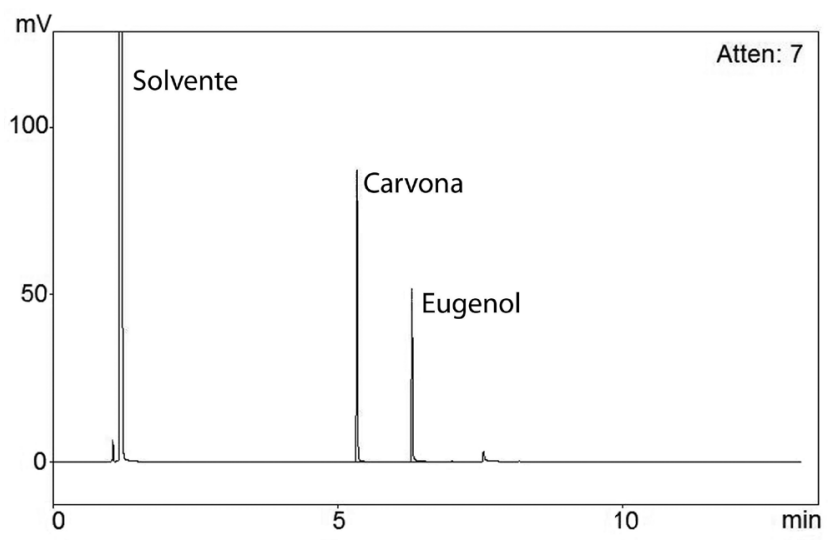

Figura 2. Cromatograma do padrão contendo eugenol e carvona

Tabela 2. Resultados das injeções dos padrões

\begin{tabular}{lccc}
\hline Padrão & Área Eugenol & Área Carvona & $\begin{array}{c}\text { Razão Áreas Eugenol/ } \\
\text { Carvona }\end{array}$ \\
\hline 1 & 33423 & 87415 & 0,382 \\
2 & 49625 & 88792 & 0,559 \\
3 & 60810 & 69865 & 0,870 \\
4 & 120861 & 102089 & 1,184 \\
5 & 176752 & 111873 & 1,580 \\
\hline
\end{tabular}

A curva de calibração direta, Figura 3, foi obtida através de um gráfico da concentração de eugenol versus a área dos respectivos sinais. O valor do coeficiente de correlação linear (R) de 0,97007, maior que o valor crítico tabelado de Pearson para $\mathrm{N}=5$ (N é o número de pontos da curva) indica uma correlação linear significativa entre os dados. Já a curva para calibração interna, Figura 4, foi construída 


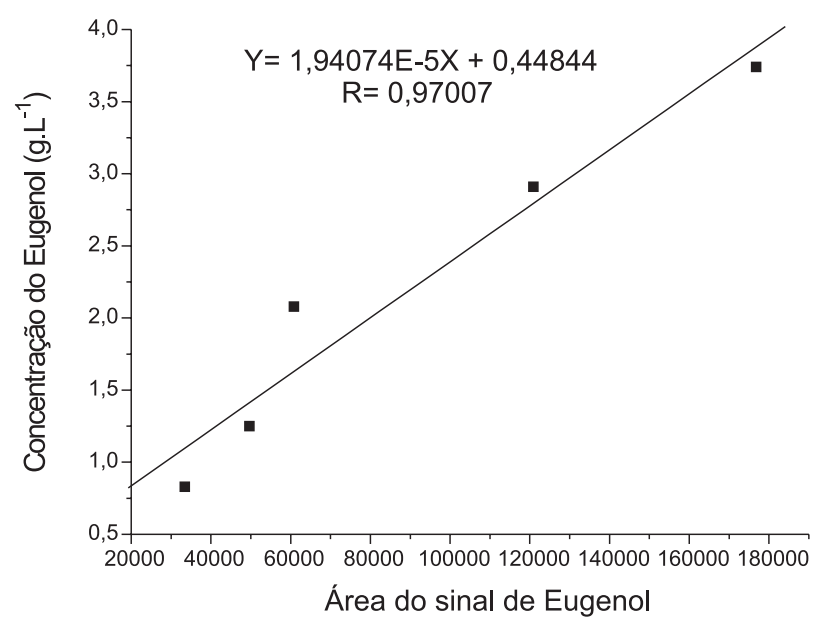

Figura 3. Curva de calibração externa do eugenol

usando a concentração de eugenol versus a razão entre as áreas dos sinais de eugenol e carvona de cada solução-padrão. $\mathrm{O}$ valor de $\mathrm{R}=$ 0,99867 mostra que a correlação linear para este caso foi mais significativa que para a curva anterior.

Idealmente as curvas de calibração deveriam passar pela origem, porém, isso não foi observado para as curvas obtidas. Desta forma, levou-se em consideração o valor dos desvios referentes a cada parâmetro das equações das retas (coeficientes lineares e angulares), fornecido pelo software utilizado na confecção dos gráficos, para avaliar a significância dos mesmos (Tabela 3). Para que o parâmetro seja estatisticamente significativo, a razão entre o valor do parâmetro e o desvio deve ser maior que o valor tabelado para o t de Student. ${ }^{8}$ Em um modelo linear, o número de graus de liberdade (GL) é dado por GL $=\mathrm{N}-2$, onde $\mathrm{N}$ é o número de pontos da curva. Para um limite de confiança de $95 \%$, o t de Student tabelado é igual a 3,182; desta forma, os valores dos coeficientes

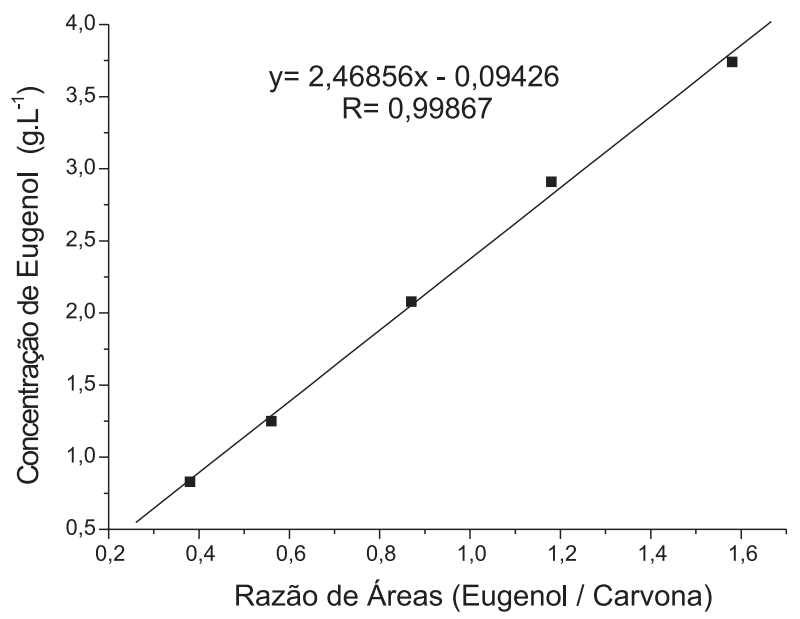

Figura 4. Curva de calibração utilizando padrão intern

lineares foram considerados estatisticamente não significativos para as duas curvas, não sendo utilizados nas equações das retas para os cálculos das concentrações.

A análise da amostra extraída do cravo-da-índia foi feita em replicata por quatro analistas, o que é um procedimento comum nos casos em que a prática é realizada por uma equipe e onde todos devem participar. A Tabela 4 mostra os resultados obtidos por cada analista levando em consideração os dois tipos de calibração.

Por análise de variância one-way dos resultados obtidos para os dois tipos de calibração, Tabela 5, foi possível constatar que as médias das duas metodologias são significativamente diferentes a um nível de confiança de $95 \% .^{9}$

Assim sendo, através desta prática foi possível demonstrar a pouca reprodutibilidade que existe nos resultados quando vários analistas estão envolvidos na injeção de uma amostra com fins quantitativos na técnica de cromatografia gasosa e, principalmente, que a utili-

Tabela 3. Análise estatística de significância dos parâmetros das curvas

\begin{tabular}{|c|c|c|c|}
\hline Parâmetros & Valores Obtidos & Desvio & t calculado \\
\hline \multicolumn{4}{|c|}{ Calibração Direta } \\
\hline Coeficiente Linear (A) & 0,44844 & 0,28912 & 1,55105 não significativo \\
\hline Coeficiente Angular (B) & $1,94074 \cdot 10^{-5}$ & $2,80483 \cdot 10^{-6}$ & 6,91928 significativo \\
\hline $\mathrm{R}$ & 0,97007 & & \\
\hline Eq. da Reta usada nos Cálculos & $Y=94074.10^{-5} \mathrm{X}$ & & \\
\hline \multicolumn{4}{|c|}{ Calibração Interna } \\
\hline Coeficiente Linear (A) & $-0,09426$ & 0,07437 & 1,26747 não significativo \\
\hline Coeficiente Angular (B) & 2,46856 & 0,07361 & 33,5357 significativo \\
\hline $\mathrm{R}$ & 0,99867 & & \\
\hline Eq. da Reta usada nos Cálculos & $Y=2,46856 \mathrm{X}$ & & \\
\hline
\end{tabular}

Tabela 4. Resultados obtidos com diferentes analistas

\begin{tabular}{lccccc}
\hline Analista & Área Eugenol & Área Carvona & $\begin{array}{c}\text { Razão Áreas } \\
\text { Eugenol/Carvona }\end{array}$ & $\begin{array}{c}\text { Cal. Dir. Conc. } \\
\text { Eugenol (g L-1) }\end{array}$ & $\begin{array}{c}\text { Cal. Int. Conc. } \\
\text { Eugenol }\left(\mathrm{g} \mathrm{L}^{-1}\right)\end{array}$ \\
\hline A & 64339 & 102144 & 0,630 & 1,249 & 1,555 \\
B & 62623 & 97877 & 0,640 & 1,215 & 1,579 \\
C & 63283 & 101065 & 0,626 & 1,228 & 1,570 \\
D & 55828 & 88254 & 0,633 & 1,083 & 1,563 \\
\hline
\end{tabular}


Tabela 5. Resultados da ANOVA para as duas calibrações

\begin{tabular}{lccccc}
\hline Fonte & GL & SQ & MQ & F & F-crítico \\
\hline Modelo & 1 & 0,278269190 & 0,278269190 & 97,31001 & 9,28 \\
Residual & 6 & 0,0171576919 & 0,00285961531 & & \\
\hline
\end{tabular}

zação de padronização interna infere um desvio muito menor nos resultados (Tabela 6). Este fato indica uma maior suscetibilidade a erros na medida de volume e de manuseio do operador do método de calibração externa. Os desvios relativos para o cálculo de eugenol na amostra com a calibração externa e interna foram de 6,272 e 0,673\%, respectivamente. O percentual de eugenol obtido no óleo através da calibração do padrão interno foi compatível com o valor apresentado na literatura (70 a $90 \%)^{3}$, enquanto que para a calibração externa o valor encontrado foi inferior ao esperado.

Tabela 6. Comparação entre as diferentes calibrações

\begin{tabular}{lcccc}
\hline Calibração & $\begin{array}{c}\text { Concentração } \\
\text { Média }\left(\mathrm{g} \mathrm{L}^{-1}\right)\end{array}$ & $\begin{array}{c}\text { Desvio } \\
\text { Padrão }\end{array}$ & $\begin{array}{c}\text { Percentual } \\
\text { de Eugenol } \\
\text { no Óleo }\end{array}$ & $\begin{array}{c}\text { Desvio Rela- } \\
\text { tivo }(\%)\end{array}$ \\
\hline Externa & 1,194 & 0,075 & 58,525 & 6,272 \\
Interna & 1,567 & 0,011 & 76,810 & 0,673 \\
\hline
\end{tabular}

Com este experimento é possível demonstrar para os alunos as diferenças entre os dois tipos de padronização, bem como fornecer a eles a oportunidade de realizar calibrações.

\section{CONCLUSÃO}

Este trabalho apresenta uma proposta de experimento empregando a cromatografia gasosa para ser utilizada em uma aula de Química Orgânica e Analítica, onde os alunos poderão aprender a realizar análises quantitativas através de calibração externa e interna. A partir do experimento proposto é possível proporcionar aos participantes a observação e assimilação dos principais fatores que influenciam uma análise quantitativa na cromatografia gasosa e as diferenças entre as calibrações, os erros que podem ocorrer, e os procedimentos necessários para minimizá-los através do uso de padrão interno.

\section{REFERÊNCIAS}

1. Skoog, D. A.; West, D. M.; Holler, F. J.; Princípios de Análise Instrumental, $5^{\text {a }}$ ed., Bookman: Porto Alegre, 2002.

2. Harris, D. C.; Análise Química Quantitativa, $5^{\text {a }}$ ed., LTC-Livros Técnicos e Científicos Editora S.A.: Rio de Janeiro, 2001.

3. Palleros, D. R.; Experimental Organic Chemistry, John Wiley \& Sons, Inc.: Nova Iorque, 1999.

4. McMurry, J.; Santos, J. P. C.; Química Orgânica, $4^{a}$ ed., LTC-Livros Técnicos e Científicos Editora S.A.: Rio de Janeiro, 1996.

5. Collins, C. H.; Braga, G. L.; Bonato, P. S.; Introdução a Métodos Cromatográficos, $7^{\mathrm{a}}$ ed., Editora da UNICAMP: Campinas, 1997.

6. McNair, H. M.; Miller, J. M.; Basic Gas Chromatography, John Wiley \& Sons, Inc.: Nova Iorque, 1998.

7. Rice, G. W.; J. Chem. Educ. 1987, 64, 1055.

8. Barros Neto, B.; Pimentel, M. F.; Araújo, M. C. U.; Quim. Nova 2002, $25,856$.

9. Miller, J. C.; Miller, J.N.; Statistics for Analytical Chemistry, $3^{\text {a }}$ ed., Ellis Horwood PRT Prentice Hall: West Sussex, 1993. 